\title{
A retrospective study of lumbar spine fusion surgery with the Wiltse approach and the traditional approach
}

\section{Weiyi Cheng ( $\nabla 20694505 @ q q . c o m$ )}

University Orthopedics https://orcid.org/0000-0001-5794-0202

\section{Xixi Zeng}

Huazhong University of Science and Technology Tongji Medical College

Tong Gui

Huazhong University of Science and Technology Tongji Medical College

\section{Research article}

Keywords: Lumbar spine fusion; The Wiltse approach; The traditional approach

Posted Date: March 24th, 2020

DOI: https://doi.org/10.21203/rs.2.22715/v2

License: (c) (i) This work is licensed under a Creative Commons Attribution 4.0 International License.

Read Full License 


\title{
A retrospective study of lumbar spine fusion surgery with the Wiltse approach and the traditional approach
}

\author{
Weiyi Cheng', Xixi Zeng', Tong Gui
}

\begin{abstract}
Background: This study aim to compare the clinical results such as functional improvement, complications, intraoperative parameters etc. between the Wiltse approach and traditional approach in lumbar spine fusion. Methods: In this retrospective controlled study, from May 2016 to May 2017, 70 patients undergone lumbar spine fusion surgery for lumbar disc herniation or lumbar spondylolisthesis within degree II were randomly divided into 2 groups which include Wiltse approach group ( $N=35, M: F=1.06: 1$, mean

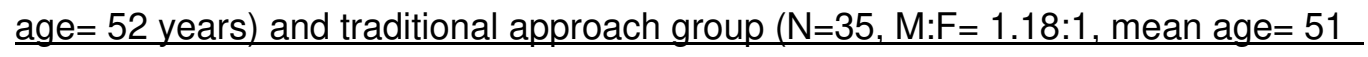
years). Actually, all the 70 cases were consisted of 38 lumbar-disc-herniation patients and 32 degree II lumbar-spondylolisthesis patients. In order to specify the clinical curative effects of the two group patients, several indicators including the operation time, the amount of blood loss and post-operation drainage, the ODI, the concentration of peripheral blood creatine phosphokinase (CK) and the cross sectional area (CSA) in magnetic resonance imaging (MRI) were compared. Results: Compared with traditional group, the Wiltse approach group had a statistically significant small figure on operative time, intraoperative blood loss, post-operative drainage and post-operative ODI $(p<$ $\underline{0.05)}$.As for the peripheral blood CK levels, the concentration of the Wiltse approach group was lower in 1 day and 3 days after the operation $(p<0.05)$, while the difference of the CK concentration for 7 days post-operation has no statistical significance $(p>0.05)$. Furthermore, for the MRI CSA three months after the operation, the Wiltse approach side's was bigger than the traditional side's in the Wiltse approach group; the Wiltse approach group's was bigger than the traditional group's of the two group's nondecompression side $(p<0.05)$. Conclusion: For lumbar spinal fusion surgery, the Wiltse approach do have such advantages as shorter operation time, smaller vertebral side muscle injury and better lumbar functional recovery compared with the traditional approach, but as a surgeon, while determining operation program, we should take into account the may influence of the inconsistency of paravertebral muscle anatomy in different levels.
\end{abstract}

Key Words: Lumbar spine fusion; The Wiltse approach; The traditional approach

Background: Lumbar spine fusion surgery is one of the commonly used surgical methods in spine surgery at present. It has wide practical value in the area of lumbar disc herniation, lumbar spinal canal stenosis, lumbar spondylolisthesis, lumbar instability and spondylolysis, vertebral body tumor and even lumbar deformity correction. The traditional 
posterior midline approach is the most common surgical approach used in lumbar spine fusion surgeries at present, but it is prone to cause the Failure Back Surgery Syndrome (FBSS)[1 ].Therefore, in 1968, Wiltse ,a surgeon, proposed the approach through the muscle space between the longissimus and the multifidus muscle, which was later modified to the current Wiltse approach. Although the Wiltse approach has gradually been adopted by surgeons in the clinical practice, evidence-based studies about it's advantages and disadvantages are still few. For this reason, we conducted a comparative study of lumbar spine fusion surgery through the Wiltse approach and the traditional posterior median approach. Following are the results.

1.Methods

1.1Participants

A total of 70 patients admitted for lumbar single-segment or double-segment fusion surgery between October 2016 and October 2017 were selected, and their clinical data were retrospectively collected. According to different surgical approaches, the patients were divided into the Wiltse approach group and the traditional approach group, with 35 cases each group. Patients including 18 male and 17 female of the Wiltse approach group were aged from 33 to 70 years with an average age of $52 \pm 11$ years old, among which the diagnosis distributions were 19 patients with lumbar disc protrusion and 16 patients with lumbar spondylolisthesis. In the traditional approach group, there were 19 male patients and 16 female patients aged from 30 to 72 years with an average age of 51 \pm 14 years old, and there are 19 cases of lumbar disc protrusion and 16 cases of lumbar spondylolisthesis. There is no statistical difference about the preoperative general clinical datum like gender, age and preoperative diagnosis of the two groups, which meant the two groups were comparable. (Table 1)

Table1 Comparison of preoperative general clinical datum of the two groups

\begin{tabular}{lccccccc} 
Group & $\mathbf{n}$ & Age(years) & BMI & \multicolumn{2}{c}{ Gender } & \multicolumn{2}{c}{ Diagnose } \\
& & & & Male & Female & LDH & LS \\
\hline WG & 35 & $52 \pm 11$ & $21.3 \pm 1.8$ & 18 & 17 & 19 & 16 \\
TG & 35 & $51 \pm 14$ & $21.6 \pm 1.5$ & 19 & 16 & 19 & 16 \\
T/X & & 0.793 & 0.815 & & 0.618 & & 0 \\
P & & 0.124 & 0.091 & 0.305 & & 1 \\
\hline
\end{tabular}

Notes: WG: Wiltse group ;TG: Traditional group; LDH: lumbar disc herniation; LS: lumbar spondylolisthesis .

Table 2 The results of general operative indexes of the two groups

\begin{tabular}{|c|c|c|c|c|}
\hline Items & $W G(n=35)$ & $T G(n=35)$ & $\mathbf{T}$ & $\mathbf{P}$ \\
\hline Operative time(mins) & $108 \pm 18$ & $134 \pm 22$ & 5.67 & $<0.05$ \\
\hline $\begin{array}{l}\text { Blood loss during } \\
\text { operation }(\mathrm{ml})\end{array}$ & $187 \pm 59$ & $389 \pm 70$ & 12.185 & $<0.05$ \\
\hline $\begin{array}{l}\text { Postoperative } \\
\text { drainage(ml) }\end{array}$ & $118 \pm 27$ & $259 \pm 62$ & 12.033 & $<0.05$ \\
\hline
\end{tabular}




\section{2 the Inclusion Criteria}

First, all patients had varying degrees of unilateral or bilateral lower limb and lumbosacral pain with obvious and severe symptoms and localizing signs. Second, after 3 months or more of conservative treatment, there was no other serious systemic disease of these patients. Third, with the help of X-ray, computerized tomography (CT) and MRI examinations of the lumbar spine, these patients were clearly diagnosed preoperatively as lumbar disc herniation, lumbar spondylolisthesis below grade II or lumbar instability requiring surgery for lumbar intervertebral disc remove and vertebral body fusion within two segments.

\section{3 the Exclusion Criteria}

Exclusion of patients whose symptoms were the results of other causes of lumbosacral pain and degeneration of the lumbar spine. Exclusion of patients with two or more lumbar segmental lesions requiring fusion surgery. Exclusion of patients with lumbar spondylolisthesis over grade II. Exclusion of patients with the height of intervertebral is too low to be inserted into the lumbar spine fusion cage. Exclusion of patients who were diagnosed as lumbar spondylolysis combined with multi-segment lumbar spinal canal stenosis or calcification of posterior longitudinal ligament. Exclusion of those with a history of lumbar spine surgery other than single lumbar spine extraction.

\section{4 the Operation Methods}

All these operations were conducted by the same surgeon team including Doctor Cheng and Gui.

\subsubsection{Wiltse approach group}

Patients of the Wiltse approach group were placed in prone position for lumbar spine surgery. Firstly, after positioning preoperative with c-arm fluoroscopy, labeling and conventional disinfection towel were performed. The intervertebral disc was treated as the central target, and the posterior midline incision was taken to expose the lumbar dorsal fascia. In the no-decompression side of vertebral plates, we pinpoint the muscle clearance of the fascia surface about 1.5 to $2 \mathrm{~cm}$ away from the midline incision, then we exposed the surface of vertebral lamina and superior as well as inferior articular process with our forefingers through the muscle clearance of multifidus muscle and longissimus. As for the decompression side, we exposed the surface of vertebral lamina and superior as well as inferior articular process by stripping the vertebral muscles. After placing pedicle screws from the herringbone ridge as the fixing point, we then used $\mathrm{C}$ arm machine to verify screws were in the right position. Next several steps were removing the decompression lateral articular process and part of the vertebral plate up and down, collecting the removed bone grain for use, cutting yellow ligament, exposing the intervertebral disc with the help of the nerve root protecting instrument, opening the fibrous ring with a sharp knife, scraping the nucleus pulposus thoroughly, opening the intervertebral space, testing the model, grafting bone into the intervertebral space, and placing a suitable sized intervertebral cage. Lastly, we had verified that the fusion cage and screws were in right position with a C-arm fluoroscopy, and then connected the vertical bar. After resetting the vertebral bodies appropriately, fixing the transverse bar and washing the wound and conducting hemostasis, we then indwelled a drainage tube 
into both decompression and non-decompression sides and sutured the incision.

\section{4 .2}

Traditional approach group

Patients of the traditional approach group were placed in prone position for lumbar spine surgery. Firstly, after positioning preoperative with c-arm fluoroscopy, labeling and conventional disinfection towel were performed. The intervertebral disc was treated as the central target, and the posterior midline incision was taken to expose the lumbar dorsal fascia. Then, as for exposing the surface of vertebral lamina and superior as well as inferior articular process, both the no-decompression and decompression sides of vertebral plates were conducted by stripping the vertebral muscles. The following steps were consistent with those of the Wiltse approach group.

\subsection{Postoperative Management}

After the operation, patients were strictly immobilized in bed for 24 hours, and intravenous antibiotics and analgesics were given for 1 day. The drainage tube was connected with the negative pressure drainage bottle, and the drainage tube was observed and recorded. The drainage tube was removed within 24-72 hours after the operation. The bed surface activity was started on the third day after the operation, waist circumference or brace activity was started on the first week, and the outpatient review was conducted within 1-3 months after the operation.

\subsection{Indicators and Methods}

The operative time (mins), intraoperative blood loss $(\mathrm{ml})$, postoperative total drainage volume $(\mathrm{ml})$ on both sides, ODI of pre-operation, 3 days and 3months post-operation, the concentration of creatine kinase (CK) in peripheral blood for 1, 3 and 7 days after surgery were recorded in detail. The CK concentration was detected by BECKMAN COULTER series AU5800 automatic biochemical analyzer. The cross-sectional area of the multifidus muscle on MRI in the adjacent segments was observed preoperatively and 3 months after the surgery. In MRI, Germany Siemens 1.5t ultra-high field superconducting magnetic resonance system was used, and t2-weighted imaging was adopted. To exclude the artifact of internal fixation from the observation level, the level near the operative segment was selected and the MRI image was transferred to the picture archiving and communication system (PACS) to outline the multifidus muscle contour by irregular curve, which was conducted by Doctor Zeng. The system automatically calculated the multifidus muscle area $\left(\mathrm{mm}^{2}\right)$.

\subsection{Statistical Treatment}

All the data are presented as mean \pm standard deviation(SD). Statistical significance was determined by Wilcoxon Rank Sum test or unpaired t test using the SPSS19.0 program (SPSS Inc., Chicago, IL, USA) with $\mathrm{P}<0.05$ being statistically significant.

\section{Results}

\subsection{Preoperative Indicators}

There is no statistically significant differences between the two groups in preoperative ODI, CK concentration in peripheral blood and MRI cross-sectional area of multifidus muscles ( $P>0.05)$, which demonstrates the two group are comparable (Table3, 4, 5, 6). 
Table 3 ODI (\%) of both groups

\begin{tabular}{lllll}
\hline ODI (\%) & WG(n=35) & TG(n=35) & $\mathbf{T}$ & $\mathbf{P}$ \\
\hline Pre the operation & $77.4 \pm 20.3$ & $75.7 \pm 22.6$ & 0.418 & 0.642 \\
\hline 3 day after the operation & $34.7 \pm 13.1$ & $44.9 \pm 14.8$ & 1.711 & $<0.05$ \\
\hline 3 months after the operation & $8.61 \pm 9.4$ & $19.42 \pm 11.3$ & 3.416 & $<0.05$ \\
\hline
\end{tabular}

Table 4 CK concentration of both groups

\begin{tabular}{|c|c|c|c|c|}
\hline $\begin{array}{l}\text { Peripheral blood CK } \\
\text { concentration (U/L) }\end{array}$ & $W G(n=35)$ & $T G(n=35)$ & $\mathbf{T}$ & $\mathbf{P}$ \\
\hline Pre the operation & $54 \pm 15$ & $55 \pm 75$ & 0.188 & 0.852 \\
\hline 1 day after the operation & $400 \pm 103$ & $598 \pm 57$ & 9.348 & $<0.05$ \\
\hline 3 days after the operation & $176 \pm 58$ & $222 \pm 50$ & 3.356 & $<0.05$ \\
\hline 7 days after the operation & $54 \pm 12$ & $57 \pm 23$ & 0.566 & 0.575 \\
\hline
\end{tabular}

Notes: the normal range of CK concentration in peripheral blood is $37-174 \mathrm{u} / \mathrm{L}$.

Table 5 The multifidus muscle cross-sectional area of the Wiltse group patients

\begin{tabular}{ccccc}
\hline $\begin{array}{c}\text { Wiltse } \\
\text { group }\end{array}$ & \multicolumn{2}{c}{$\begin{array}{c}\text { Cross-sectional area }\left(\mathbf{m m}^{2}\right) \\
\text { non-decompression side }\end{array}$} & $\mathbf{T}$ & $\mathbf{P}$ \\
\hline $\begin{array}{c}\text { Pre the } \\
\text { operation }\end{array}$ & $424 \pm 11$ & $424 \pm 10$ & 0 & 1.0 \\
$\begin{array}{c}3 \text { months } \\
\text { after the }\end{array}$ & $347 \pm 73$ & $259 \pm 82$ & 16.986 & $<0.05$ \\
operation & & & & \\
\hline
\end{tabular}

Notes: In the Wiltse approach group, the non-decompression side was the Wiltse approach side, and the decompression side was the traditional approach side.

Table 6 The cross-sectional area of the multifidus muscle in both groups

\begin{tabular}{ccccc}
\hline $\begin{array}{c}\text { Non- } \\
\text { decompression } \\
\text { side }\end{array}$ & $\begin{array}{c}\text { Cross-sectional area }\left(\mathbf{m m}^{2}\right) \\
\text { Wiltse group }\end{array}$ & Traditional group & & T \\
\hline $\begin{array}{c}\text { Pre the } \\
\text { operation }\end{array}$ & $424 \pm 11$ & $428 \pm 82$ & 0.255 & 0.8 \\
$\begin{array}{c}\text { 3 months after } \\
\text { the operation }\end{array}$ & $347 \pm 73$ & $239 \pm 78$ & 6.482 & $<0.05$ \\
\hline
\end{tabular}

\subsection{General Postoperative Indicators}

There are statistically significant differences between the two groups in operation time, intraoperative blood loss and postoperative drainage volume $(\mathrm{P}<0.05)$ (Table 2).

2.3 The Oswestry Disability Index (ODI)

The postoperative ODI differences of the two groups for 3 days and 3 months are statistically significant $(\mathrm{P}<0.05)$ (Table3).

2.4 Postoperative Peripheral Blood CK Concentration

The difference of $\mathrm{CK}$ concentration in peripheral blood between the two groups is statistically significant for 1 and 3 days after the surgery $(P<0.05)$. There is no significant 
difference in CK concentration in peripheral blood for 7 days after the surgery between the two groups $(P>0.05)$ (Table 4).

\section{$2.5 \mathrm{MRI}$ Results}

2.5.1 For patients of the Wiltse approach group, there is no statistically significant difference in the cross-sectional area of the multifidus muscle on preoperative MRI between the decompression side and compression side $(P>0.05)$, but on three months, MRI after the surgery, the difference is statistically significant $(P<0.05)$. (Table 5) 2.5.2 When comparing the non-decompression side of the two groups patients, there is no statistically significant difference in the cross-sectional area of the multifidus muscle on preoperative MRI $(P>0.05)$. The difference in MRI cross-sectional area of the multifidus is statistically significant three months after the surgery $(P<0.05)$. (Table 6 and Figure1)

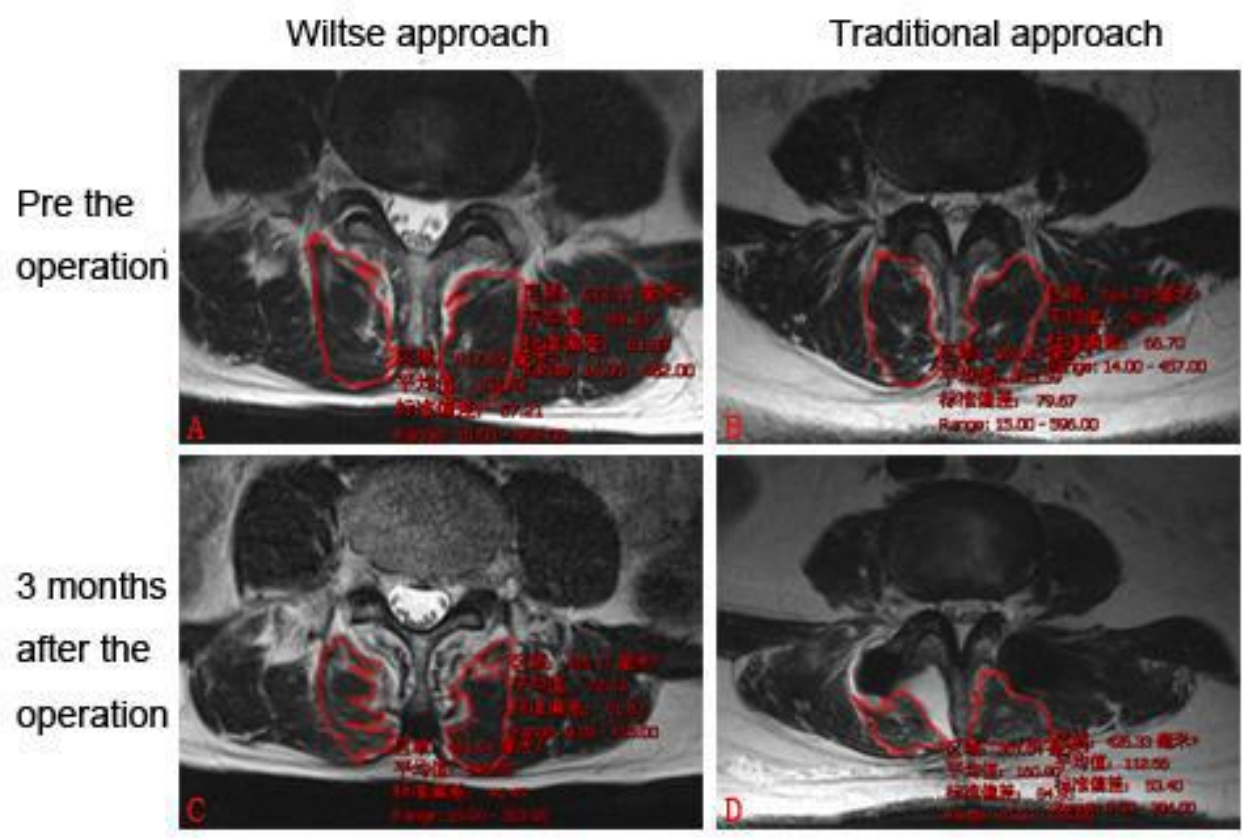

Note: $A$ and $C$ show the cross-sectional area of multifidus muscle on MRI for L3/L4 level of a 38-year-old male LDH patient who underwent lumbar spine fusion operation with the conventional approach; B and D show the cross-sectional area of multifidus muscle on MRI for L3/L4 level of a 56-year-old female LDH patient who underwent lumbar spine fusion operation with the Wiltse approach.

Figure1 The cross-sectional area of multifidus muscle of two different surgical approach patients on MRI before and after surgery were observed

\section{Discussions}

\subsection{Advantages of intermuscular approach in lumbar spine fusion}

3.1.1 Less intraoperative hemorrhage is beneficial to obtain a clear surgical field and save the operation time. The blood supply to the paravertebral muscles is unilateral circulation, there is no traffic branch between the muscles, and there is no obvious cross vessel between the multifidus muscle and the longest muscle. Therefore, the operation on the physiological interspace between the multifidus muscle and the longest muscle can reduce the amount of intraoperative blood loss, greatly reducing the duration of 
hemostasis in the operation and making the operative field relatively clear. The results of this study showed that, compared with the traditional dissection approach, the amount of intraoperative blood loss and postoperative drainage fluid is less, which is consistent with the anatomy and physiology. In this investigation, the operation time of the two groups of patients is compared, and the results shows that the operation time of the Wiltse approach group is significantly less than that of the traditional approach group, which may be directly related to less intraoperative bleeding and clear intraoperative vision. 3.1.2 Less damage to the multifidus. The traditional approach requires extensive dissection of the paravertebral multifidus muscle, which will damage the blood supply and nerve innervation of the muscle, and a series of degeneration phenomena such as muscle atrophy, fibrosis and fat accumulation will occur in the multifidus muscle postoperatively [2]. The direct result is that the normal physiological function of the multifidus is affected postoperatively, and the incidence of postoperative chronic low back pain is increased [3]. The Wiltse approach is operated through the normal physiological muscle space, which does not require extensive paravertebral muscle stripping, so it raises less damage to the multifidus muscle and leads to a better lumbar functional recovery demonstrated as the ODI results of this study.

In order to compare the degree of paravertebral muscle injury caused by the two approaches, we compare the postoperative peripheral blood CK concentrations of the two groups of patients. Kawaguchi et al. [4] proved that CK concentration in peripheral blood increased with paravertebral muscle injury. Lombao Iglesias D et al. [5] proved that $\mathrm{CK}$ concentration in peripheral blood began to increase after surgery, returned to normal within 1 week, and was positively correlated with the degree of muscle injury. In this study, the difference of CK concentration in peripheral blood between the two groups is statistically significant for 1 day and 3 days after surgery, indicating that the muscle gap approach is less severe for paravertebral muscle injury. The CK concentration returned to normal level 7 days after surgery, and the results of this experiment are consistent with the results of the above study.

Zhi Jun $\mathrm{H}$ et al. [6] showed that magnetic resonance imaging is an accurate method to evaluate the injury and atrophy of multifidus muscles. In order to determine the quantitative difference between the two approaches for multifidus muscle injury, this study conducted a statistical analysis of the MRI transversal area of multifidus muscle three months after the operation between the two groups. In the Wiltse approach group, the Wiltse approach was performed on the non-decompression side, and traditional approach was performed on the decompression side. Statistical analysis was performed on both sides. The results showed that the preoperative difference in cross-sectional area of bilateral multi-fissure muscles was $p>0.05$, which was not statistically significant. Three months after the operation, the difference in the cross-sectional area of the multifidus muscle on both sides was $p<0.05$, which was statistically significant. The nondecompression side of the muscle gap approach group was compared with the nondecompression side of the traditional approach group, and the preoperative difference in the cross-sectional area of the multifidus muscle was $p>0.05$, which was not statistically significant. Three months after the surgery, the change in cross-sectional area of multifidus muscle was statistically significant $(p<0.05)$. These results show that the Wiltse 
approach resulted in a reduction in loss of the cross-sectional area of multifidus muscle, indicating that the Wiltse approach has less damage to multifidus muscle. These findings were consistent with multiple studies $[7,8,9,10]$.

3.2 The insufficiency of the Wiltse approach in lumbar spine fusion surgery.

The paravertebral muscles are not completely parallel to the spinous process of the spine, and the lower lumbar paravertebral muscles are relatively bulky and deviates from the median line [11]. Therefore, in the upper lumbar spine, surgery was performed through the paravertebral space to facilitate the exposure of the lumbar facet and transverse process, which is conducive to pedicle screw placement. In the lower lumbar spine, the Wiltse gap is far away from the midline structure, and the intraoperative operation Angle is large, which is conducive to the exposure of the intervertebral foramen and the side of the lumbar spine, and is convenient for the removal of the extremely lateral intervertebral disc. However, the poor display of the vertebral canal structure is not conducive to the decompression of nerve roots [12]. Therefore, in lumbar spine fusion surgery, it should be fully understood that the anatomical position difference of the Wiltse gap at different levels will affect the operation.

\section{Conclusions}

For lumbar spinal fusion surgery, the Wiltse approach do have such advantages as shorter operation time, smaller vertebral side muscle injury and better lumbar functional recovery compared with the traditional approach, but as a surgeon, while determining operation program, we should take into account the may influence of the inconsistency of paravertebral muscle anatomy in different levels.

5.Declarations

Ethics approval and consent to participate

This study was supported by the ethic committee of Maternal and Child Hospital of Hubei Province. Informed consent was waived by the committee because of the retrospective nature of the study.

Consent for publication

Not applicable.

Availability of data and materials

The datasets used and/or analyzed during the current study are available from the corresponding authors on reasonable request.

Competing interests

The authors declare that they have no competing interests.

Funding

Not applicable. 
Authors' contributions

Weiyi Cheng and Xixi Zeng conceived and coordinated the study, designed, performed, and analyzed the experiments, and wrote the paper. Weiyi Cheng ,Xixi Zeng and Tong Gui carried out the data collection, data analysis, and revised the paper. All authors reviewed the results and approved the final version of the manuscript.

Acknowledgements

Not applicable.

\section{Abbreviations}

CK: creatine phosphokinase

CSA: cross sectional area

MRI: magnetic resonance imaging

FBSS: Failure Back Surgery Syndrome

LDH: lumbar disc herniation

LS: lumbar spondylolisthesis

CT: computerized tomography

PACS: picture archiving and communication system

\section{References}

1.Al Kaisy A, Pang D, Desai MJ, et al. Failed back surgery syndrome: who has failed?. Neurochirurgie. 2015;61 Suppl 1:S6-S14. doi:10.1016/j.neuchi.2014.10.107. 2.Street JT, Glennie RA, Dea N, et al. A comparison of the Wiltse versus midline approaches in degenerative conditions of the lumbar spine [J]. J Neurosurg Spine,2016,25(3):332-338.

3.LIU Z, LI Z, XING D, et al. Two different surgery approaches for treatment of thoracolumbar fracture [J] .Int J Clin Exp Med,2015,8 (12): 22425-22429. 4.Kawaguchi $\mathrm{Y}$, Matsui $\mathrm{H}$, Tsuji $\mathrm{H}$. Changes in serum creatine phosphokinase MM isoenzyme after lumbar spine surgery. Spine (Phila Pa 1976), 1997, 22:1018-1023. 5.Lombao Iglesias D, BagóGranell J, Vilor Rivero T. Validity of creatine kinase as an indicator of muscle injury in spine surgery and its relation with postoperative pain. Acta Orthop Belg. 2014;80(4):545-550.

6.ZhiJun $\mathrm{H}$, WenBin $\mathrm{X}$, Shuai $\mathrm{C}$, et al. Accuracy of magnetic resonance imaging signal intensity ratio measurements in the evaluation of multifidus muscle injury and atrophy relative to that of histological examinations. Spine. 2014 May 1;39(10):E623-629.

7.Zhou C, Tian Y, Zheng Y, et al. Mini-invasive transforaminal lumbar interbody fusion through Wiltse approach to treating lumbar spondylolisthesis. Orthop Surg. 2016; 8:4450 .

8.Li B, Wu ZG, Peng XZ, et al. Lumbar discectomy via Wiltse approach. Orthop Surg. 2014; 6:80.

9. Ulutas, M, Yaldız C, Seçer M, et al. Comparison of Wiltse and classical methods in surgery of lumbar spinal stenosis and spondylolisthesis. Neurol Neurochir Pol. 2015; 
49:251-257.

10.Warren A, Prasad V, Thomas M. Pre-operative planning when using the Wiltse approach to the lumbar spine. Ann R Coll Surg Engl. 2010; 92:74-75.

11. Fei Yang, Wei Lu, Yonggang Wu. Comparison of the Wiltse approach and traditional approach in the treatment of thoracolumbar fracture [J]. Chinese journal of orthopaedic surgery.2018,26(10):903-908.

12.Shi-dong Wang, Xue-fei Deng, Zong-sheng Yin, Wei Wang, Yong Hu, Youzhi Zhu, Yu Zhang, Hui Han. Anatomical and imaging observation of posterior approach of lumbar paravertebral space [J]. Chinese journal of spinal cord and spinal cord. 2013,23(03):257262.

Authors'information

Affiliations

Department of Orthopedics, Maternal and Child Hospital of Hubei Province, Tongji Medical College, Huazhong University of Science and Technology, Wuhan, Hubei 430000, China. Weiyi Cheng and Tong Gui

Department of Anatomy, Tongji Medical College,Huazhong University of Science and Technology, Wuhan, Hubei 430000, China. Xixi Zeng

Corresponding author

Correspondence to Weiyi Cheng. 


\section{Figures}

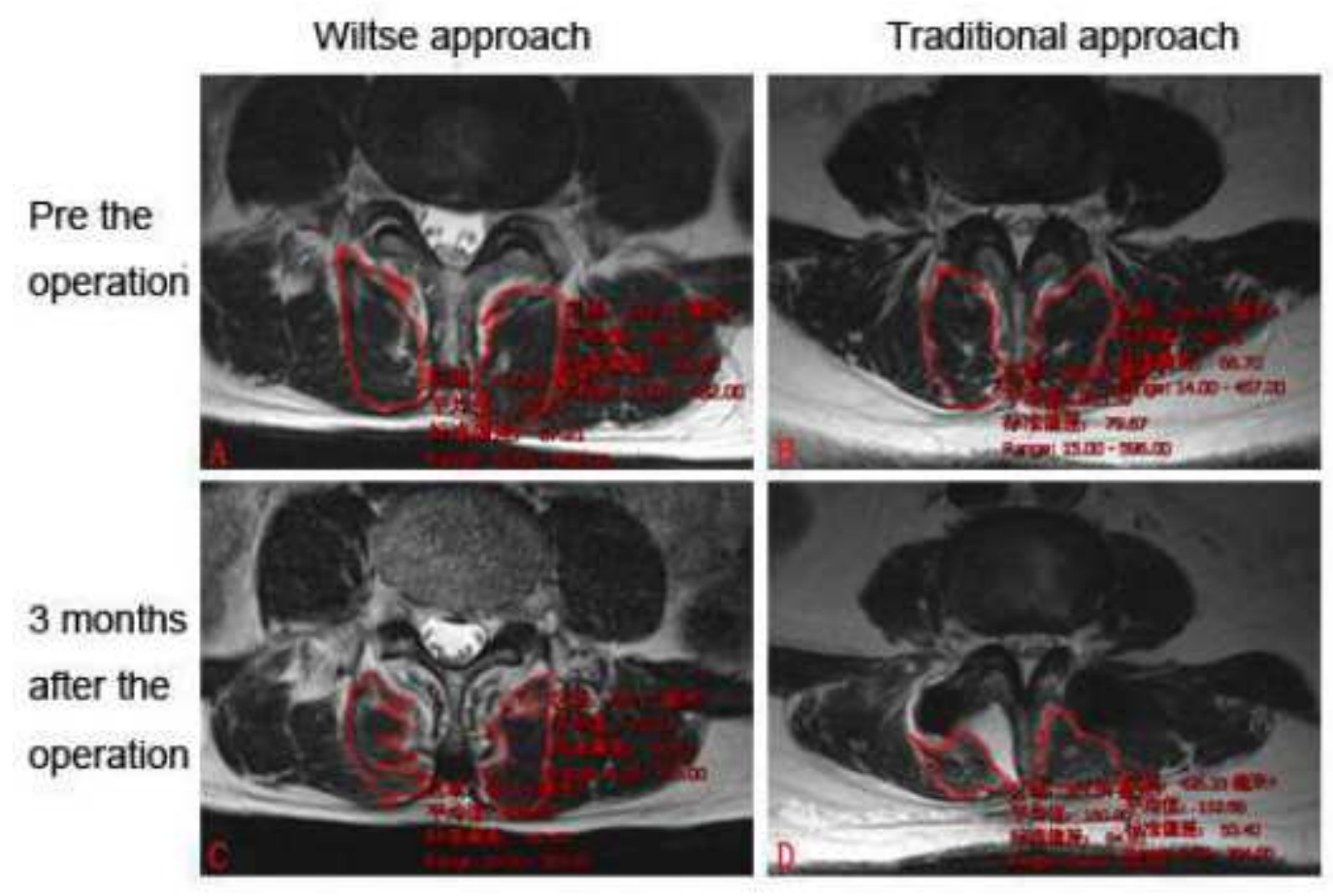

\section{Figure 1}

The cross-sectional area of multifidus muscle of two different surgical approach patients on MRI before and after surgery were observed. Note: $\mathrm{A}$ and $\mathrm{C}$ show the cross-sectional area of multifidus muscle on MRI for L3/L4 level of a 38-year-old male LDH patient who underwent lumbar spine fusion operation with the conventional approach; $B$ and $D$ show the cross-sectional area of multifidus muscle on MRI for L3/L4 level of a 56-year-old female LDH patient who underwent lumbar spine fusion operation with the Wiltse approach. 